\title{
Bit noise from an optical logic gate with laser diodes
}

\author{
Ana P. González-Marcos ${ }^{1}$, José A. Martín-Pereda ${ }^{2}$, A. Hurtado $^{3}$ \\ E.T.S. Ingenieros de Telecomunicación. Universidad Politécnica de Madrid \\ Ciudad Universitaria. 28040 Madrid. Spain
}

\begin{abstract}
Output bits from an optical logic cell present noise due to the type of technique used to obtain the Boolean functions of two input data bits. We have simulated the behavior of an optically programmable logic cell working with Fabry Perotlaser diodes of the same type employed in optical communications $(1550 \mathrm{~nm})$ but working here as amplifiers. We will report in this paper a study of the bit noise generated from the optical non-linearity process allowing the Boolean function operation of two optical input data signals. Two types of optical logic cells will be analyzed. Firstly, a classical "on-off" behavior, with transmission operation of LD amplifier and, secondly, a more complicated configuration with two LD amplifiers, one working on transmission and the other one in reflection mode. This last configuration has nonlinear behavior emulating SEED-like properties. In both cases, depending on the value of a "1" input data signals to be processed, a different logic function can be obtained. Also a CW signal, known as control signal, may be apply to fix the type of logic function. The signal to noise ratio will be analyzed for different parameters, as wavelength signals and the hysteresis cycles regions associated to the device, in relation with the signals power level applied. With this study we will try to obtain a better understanding of the possible effects present on an optical logic gate with Laser Diodes.
\end{abstract}

Keywords: Optical-noise, semiconductor-lasers, optical-bistability, optical computing, logic cell.

\section{INTRODUCCION}

The subject of Boolean functions can be associated, in a first view, with its application on Digital Computing field. But they are also applied in networks on switching or routing duties [1]. The main difference, with respect to their use, between computer and communication fields is related with the signal degradation originated during transmission, being much more important in the second case than in the first one. In any case, the concept of being able to recognize the right signal is of fundamental importance in both case. In both cases, it is necessary to analyze a black box where it is introduced an ideal signal in the input and, theoretically, on the output we should have another ideal signal; in communication it is desired to have the same signal at the input that at the output. Moreover, in computer or processing signals (switching or routing) the output must be an ideal function of the input. Taking into account that it is impossible to obtain a sharp digital pulse and being aware that time dependent analysis must be done with a further accuracy and, moreover, that it is necessary to fully understand the behavior in order to control the results, we present in this work an approached to the evaluation of a digital logic cell with a classical technique used to validate a digital communication link, namely, with the well known "eye diagram" [2]. Ads it may be seen in any text book, this diagram it gives the values of noise in a bit ' 1 ' and in a bit ' 0 ' and, at the same time, it represents too the time response on initial and final slopes of the signals. Namely, the way the eye is open means how separated are the two power or voltage levels, for " 0 " and " 1 ", and allows us to evaluate how easy can be to regenerated the initial signals.

On computing [3- 4], the analysis of signals by the method of the "eye diagram" has never been employed. The handling of binary signals is mostly related here with the possibility to implement logical functions and the possible errors had during the computation. Noise, as a fundamental part of design, has not had a fundamental role. But coarsely, noise can be viewed as the random deviation of a physical parameter from an expected value. Considering light, this physical parameter can be for instance: the electric field amplitude, the optical power, the frequency, the phase or the polarization. Any one of them has a significant influence on the final result. This aspect has been considered in several

1 agonmar@tfo.upm.es

2 jamp@tfo.upm.es

${ }^{3}$ hurtado@tfo.upm.es 
occasions due to the importance of small deviations on the signals with respect to their correct positions. These deviations may be due to small changes in the devices characteristics or due, as it is now the case to be analyzed, to the presence of some noise added to the signal. Moreover, as it is known, noise can be described from two different points of view. One is the classical (or semi classical) description. The second one is the quantum approach. This quantum analysis is not really used in engineering as it does not have to be concerned so much with the origin of physical fundamentals. [5]

It is because that, we have adopted a different approach to the present case. If we want to analyze the influence of noise on the performance of digital architectures we must take into account how these architectures are going to be employed in real structures and under real conditions. Noise will be always present. But the more important fact will be no the origin of the noise. The fact that will be of importance is its influence on the final behavior of the system. And it is because that, we have taken some concepts from optical communications, where this noise is analyzed with different method to the usually employed in more basic situations. In this way, the concept of bit-error-rate is central to digital communications engineering. The BER represents the symbol-averaged probability of transmission failure, meaning that such a probability should be brought under an acceptable reference level. This concept has not been employed in optical computing and it will be used here.

In these work we will present first the structure employed to obtain optic logic gates in order to obtain the different logic Boolean functions. As in every one of the cases the cell will be based in semiconductor laser diode structures, the fundamental and already known effects that noise origin will be enumerated. Finally the previous structures have been simulated with a computer program and the results, on terms of eye diagrams, will be discussed.

\section{LASER DIODES AS OPTICAL LOGIC GATE - OLG}

Optical bistability in laser diode amplifiers has been in the last years an intensive research topic due to the potential applications of these devices in different fields of technology, and for their practical advantages of laser amplifiers: the presence of gain, fast response, low optical power requirements to achieve bistability etc.

The physical phenomenon that causes the optical bistability in laser amplifiers is the dispersive nonlinearity, characterized by the existence of a resonator filled with a medium whose refractive index depends on the incident optical power. An increment in the optical power injected into a laser makes its resonant frequency to move towards longer wavelengths, due to the variation of the refractive index. This means that when an optical signal, detuned to the long wavelength side of the resonant frequency mode of the laser is injected into it, will cause, as a consequence of the variation of the refractive index of the active medium, the positive wavelength shift of the resonant emission frequency of the laser. Once a threshold is achieved the optical bistability can be observed. For a more detailed explanation of the optical bistability in laser amplifiers see for example [6]

\subsection{Structures and configurations}

Several can be the configuration in order to obtain a logic gate. In our case the model is based in the basic devices that conform an OPLC - " Optical Programmable Logic Cell" already publicized and well described in [7].The OPLC allows all the Boolean functions giving two outputs for one pair of digital data inputs. The basic devices are two, we call them optical logic gate and we will analyze the bit noise associated for both of them. First and deeper we will analyze the more simpler device denominated Q-device, which behavior is an on-off function many times implemented for different applications. Second a brief detail of a more complex device, the P-device, will be presented. The transfer function optical input-output power of the OPG used in section 4 is described here for different structures.

In all the configurations the input data is sum in such a way that always there is a multilevel signal to be processed inside the device.

\subsubsection{Q-device}

In this section we will describe briefly how is implemented the device. We have used two types of laser diode in order to obtain bistability: a Fabry-Perot and a DFB structure. Figure 1 shows the block diagram with 3 input signal, two for data and for a CW control signal that's allows the programmable of the logic function. Also in figure 1 can be seen the transfer function for the two types of structure. They have been simulated on the way that input optical power for data bit ' 1 ' should be the same. This correspond to the same power level for the device to switch (around $70 \mu \mathrm{W}$ ) for both device. As it can be seen the output optical power is higher for the DFB-LD. 


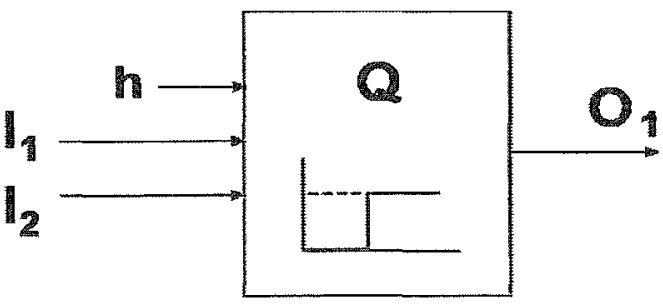

\begin{tabular}{|l|l|l|l|}
\cline { 2 - 4 } \multicolumn{1}{c|}{} & $\mathrm{h}_{0}$ & $\mathrm{~h}_{1}$ & $\mathrm{~h}_{2}$ \\
\hline $\mathrm{O}_{2}$ & $\mathrm{AND}$ & $\mathrm{OR}$ & $\mathrm{ON}$ \\
\hline
\end{tabular}
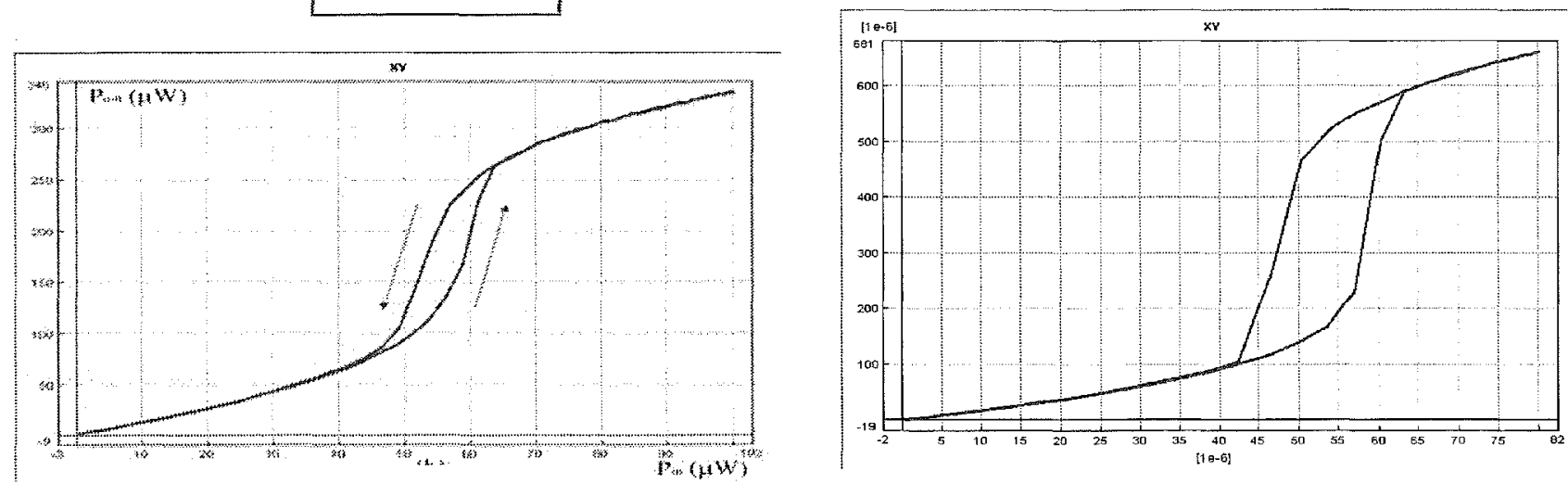

Figure 1. - Block Diagram a Q-device. Logic Table for the OLG. Left side optical output power Pout characteristic in function of optical input power Pin for a Fabry-Perot Laser Diode. Right side for a DFB Laser Diode.

\subsubsection{P-device}

The P-device was conceived as SEED device; in order two obtain the same transfer function the design structure consists on two coupled FP Laser Amplifiers the first laser is operated in reflection, while the second is operated in transmission. The first laser performs the basic bulk of the logic processing, while the second acts as an optical power shareholding limiter. For a deeper description see [6]. Figure 2 shows all the information about this device.
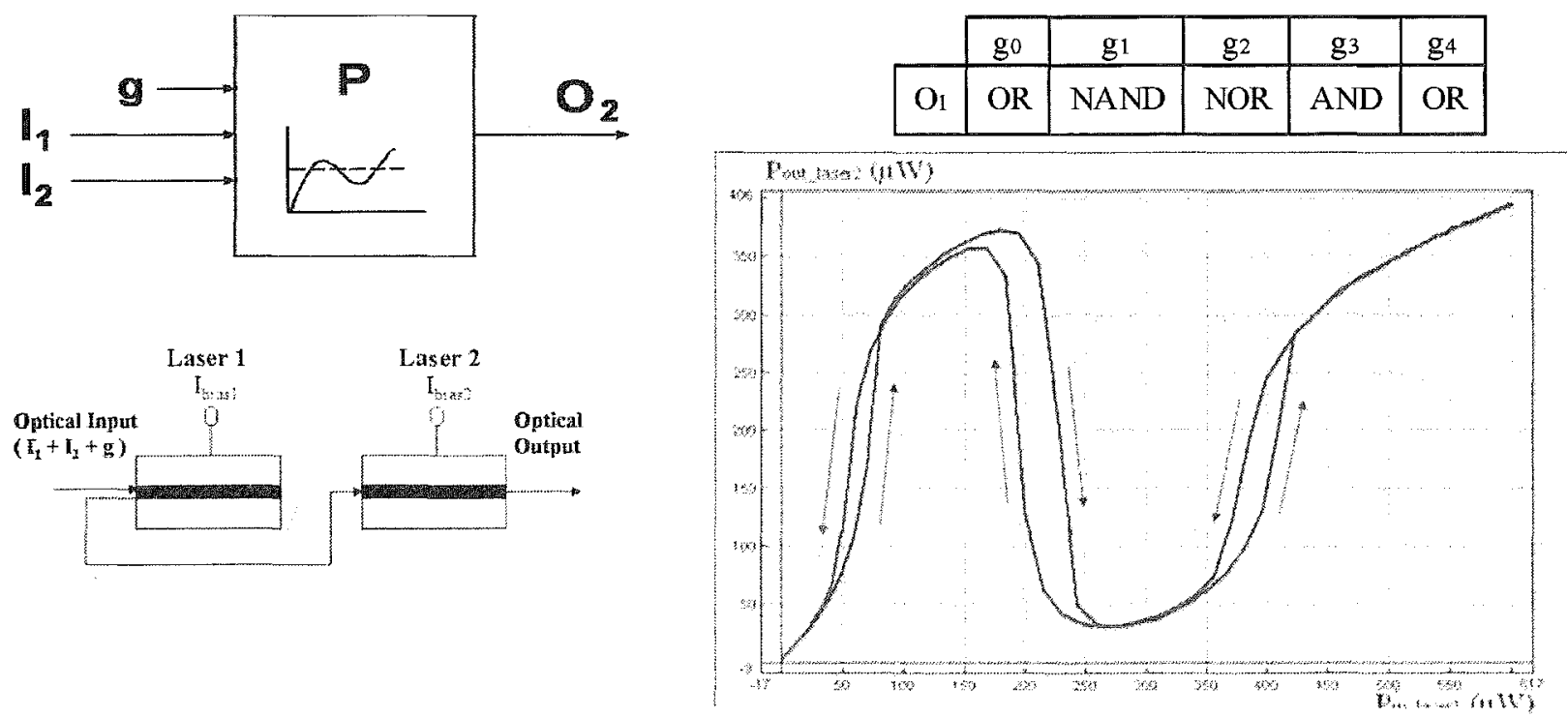

Figure 2. - Block Diagram of P-Device. Logic table for OLG. Laser diode configuration and optical output power Pout characteristic in function of optical input power Pin for FP-LD configuration. 


\section{Noise Analyzed in Laser Diodes as OLG}

Dynamic properties of optically switched semiconductors lasers biased from below-to-above threshold have been already study theoretically in several places. In reference 8 there is a review and discussion of principal's dynamic properties until the publications date.

Some studies have been done on a time-dependent theory of optical bistability. The best well know is the one development by M.J.Adams [9]. In his model is analyzing that in a Fabry-Perot (FP) cavity there is an unavoidable feature of bistable action: the presence of an output intensity spike, as the phase must always past through a resonance during switch-up (and switch -down). This affect from a practical point of view mainly in timing as due to it there is an associated time delay before steady-state output is achieved. This spike can be seen in our simulation.

Also similar studies have been done to the case of Distributed Feedback (DFB) structures exploring the potential of optical bistability in DFB amplifiers both theoretically and experimentally [10-12]. The optical bistability is observed at input laser levels of a few $\mu \mathrm{W}$ and also appears a pronounced spike in the output transient response form the device.

With respect to the noise at the different power levels, in next section is demonstrated that an FP- laser diode has more noise due to the presence of a multimode longitudinal response of laser which feedback as obtained with a FP cavity. The DFB structure used for the simulation has only one longitudinal mode so noise has an small level.

\section{Bit noise in OLG with Laser Diodes.}

We are not trying to understand the origin of noise. We just compare different configurations in order to see which one gives a better behavior. With an eye pattern there is enough information about the performance of a data transmission or in this case of a data processing. Eye pattern is defined as the synchronized superposition of all possible realizations of the signal of interest. The width of the eye opening defines the time interval over which it can vary the timing error and the height of the eye opening, at a specific sampling time, defines the noise margin of the system or device.

In the case of M-ary system, the eye pattern contains (M-1) eyes openings stacked up vertically one on the other, where $\mathrm{M}$ is the number of discrete amplitude levels used to construct the transmitted signal. In our case there are two eye opening but one is very small compared to the other so is negligible.

In order to obtain a clear eye pattern must be used a random input signal. In all figures is represented the input signal applied in each case study. We have not presented a longer signal in time because it takes a lot of time to simulate the response of the laser amplifiers. It is necessary a high band width to take in account the most number of longitudinal modes of the laser cavities.

We compare two different structures for a same type of device and function; and secondly we shows the results for two functions, AND and OR, obtained from a different configuration or device.

\subsection{Simulation results $\mathrm{DFB}$ and $\mathrm{FP}$-cavity comparison}

First it will be study the behavior of Q-device, which has a simpler configuration as it was explained in section

2. The way to know how much noise is introduced by the logic gate in a bit is analyzed by a scheme, modeled taking use of the VPI ComponentMaker ${ }^{T M}$ software tool. Main parameters of simulation are related on the table at the end of this document. The table has the parameter values for two types of FP and DFB. In Q-device model it has been used FP1 and DFB internal structure of laser amplifiers. As can be see on the table the amplifier are biased below threshold

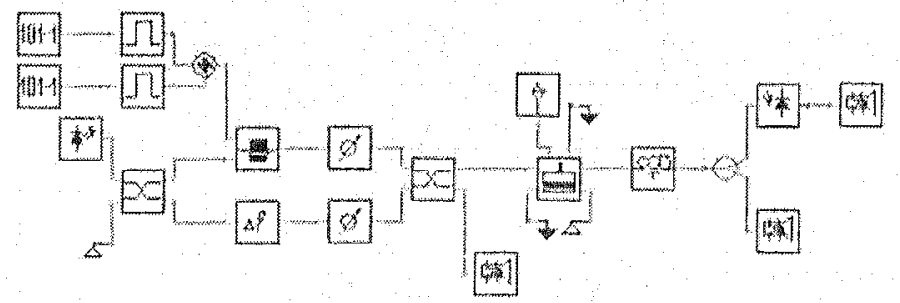

Figure 3. - Schematic of the model simulated by VPI_ComponentMaker ${ }^{\mathrm{TM}}$ software tool for Q-device. current. The eye pattern has been obtained for an specific condition of laser diode and optical signal used for the data. Any change on these parameters will produce different results [9].

Figure 4 shows all the representative information related to the function $O R$, which is obtained with a Q-device with a CW control signal equal to zero. The details of the transition that affect more to the noise present on the eye pattern are also represented. As it can be seen DFB structure has less noise but the spike is higher. The spike affect to the time response of the device. Our study is made 
with a $100 \mathrm{Mbps}$ input data signals. The conclusions obtained from the eye pattern are restricted to this signal. If we change the rate a new representation must be done and other conclusions will be extracted. In any case deeper study gives information about in what part of the transfer function must have the input level in order to have less noise or time dependence. As we see in figure 4 transition from 1-0, or 0-1 is not relevant.
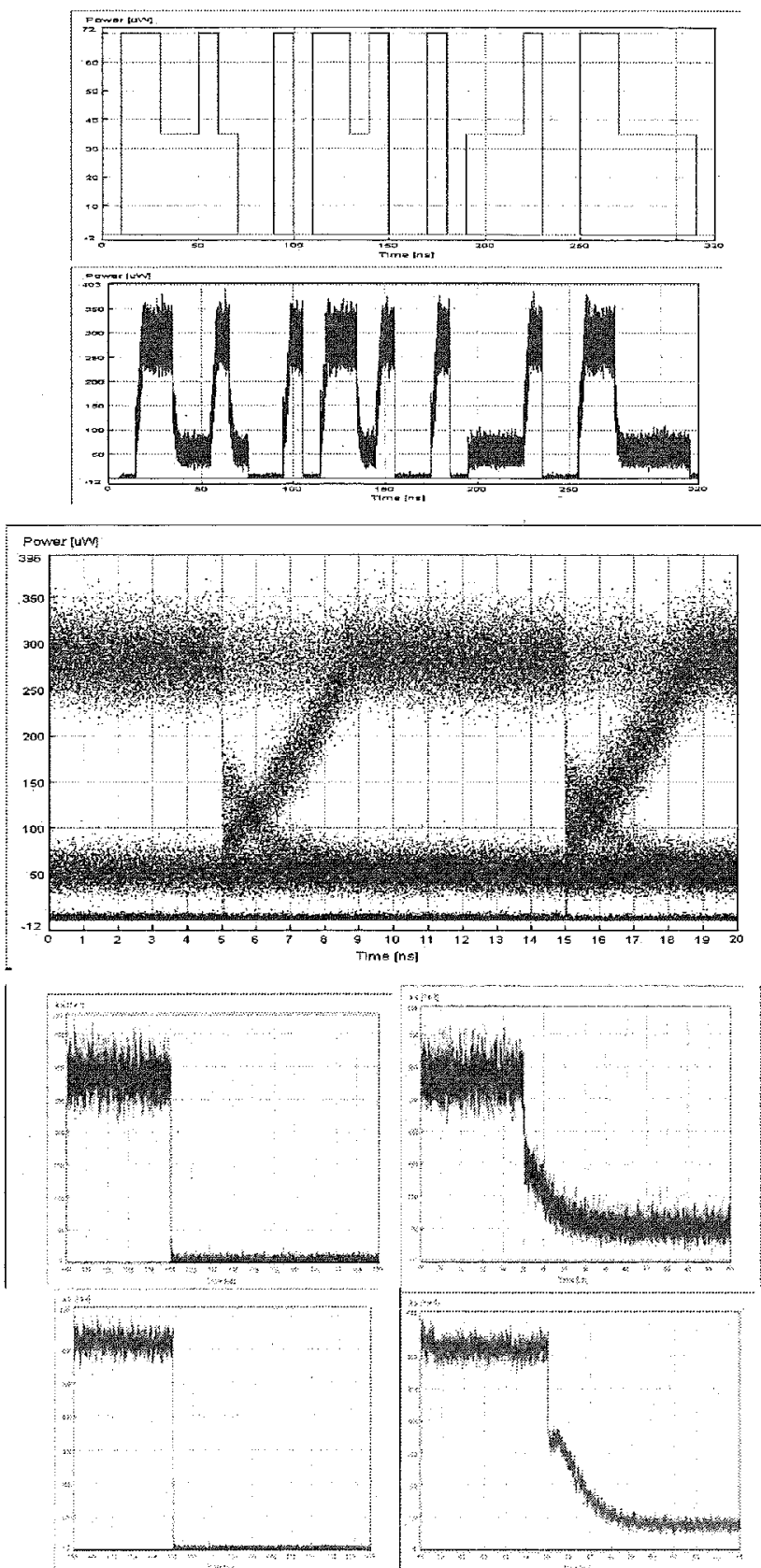
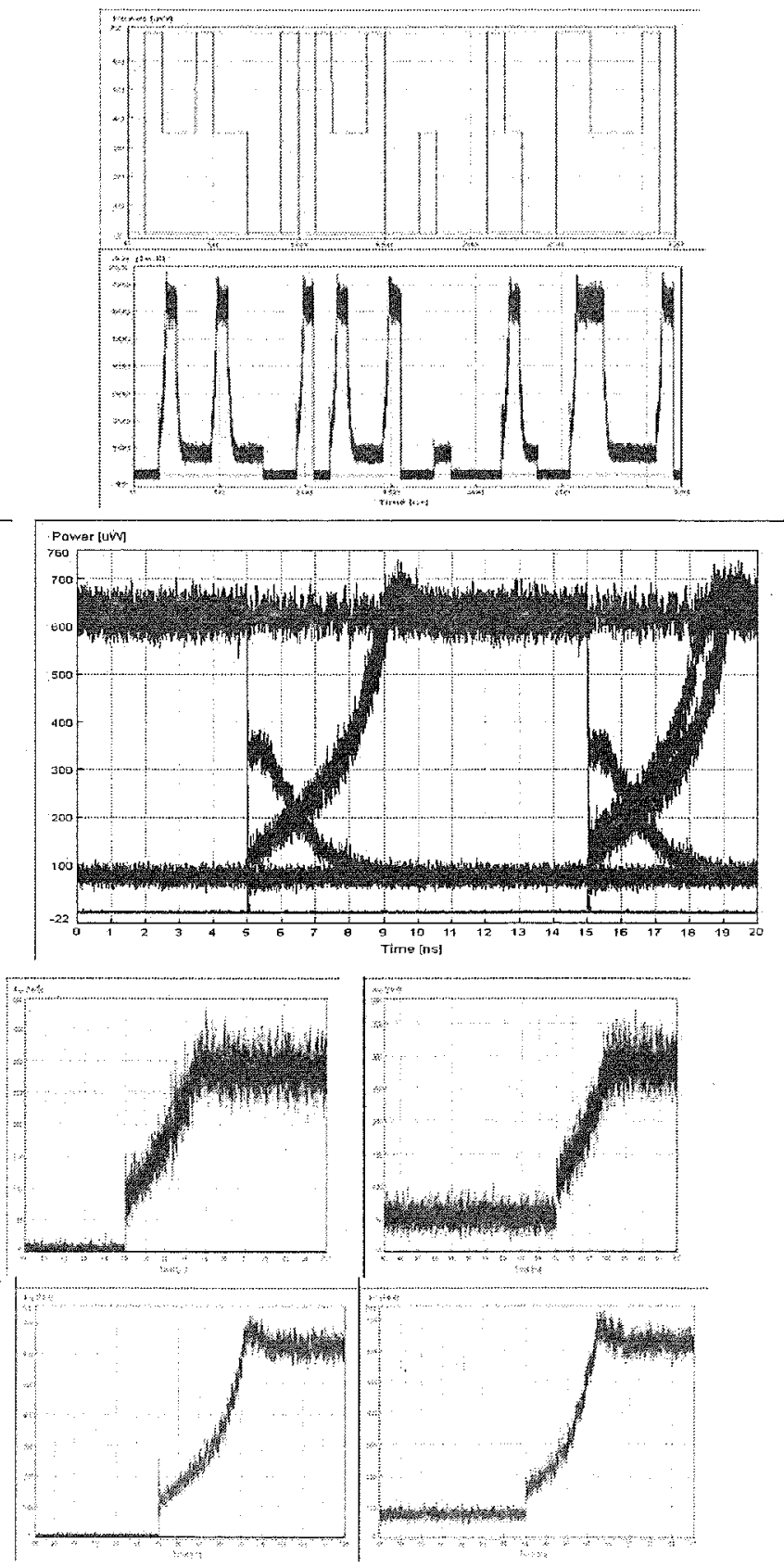

Figure 4. - First line of graphs shows the input data to the OLG. Second line the output of an AND function for the corresponding input data. Third line the eye diagram representation. Left column is for Q-device structure in Fabry-Perot and right column for DFB.

Two last lines: the one with higher noise correspond to FP_LD; last line to the DFB LD. The detail representations of significantly transition are show: transition of input data from $2-0,2-1,0-2, \overline{1}-2$ 
AND function is analyzed on figure 5. The input signal is represented separately because the random signals change from one simulation to another. With FP-LD there is a different signal that with DFB but the eye pattern in both cases is the same. It is not represented the entire eye pattern due to the time simulation required as it was explained before. With this function the noise is higher and time respond is worst because the $\mathrm{CW}$ control signal that force the device to execute AND function force to work very near of the hysteresis cycle.
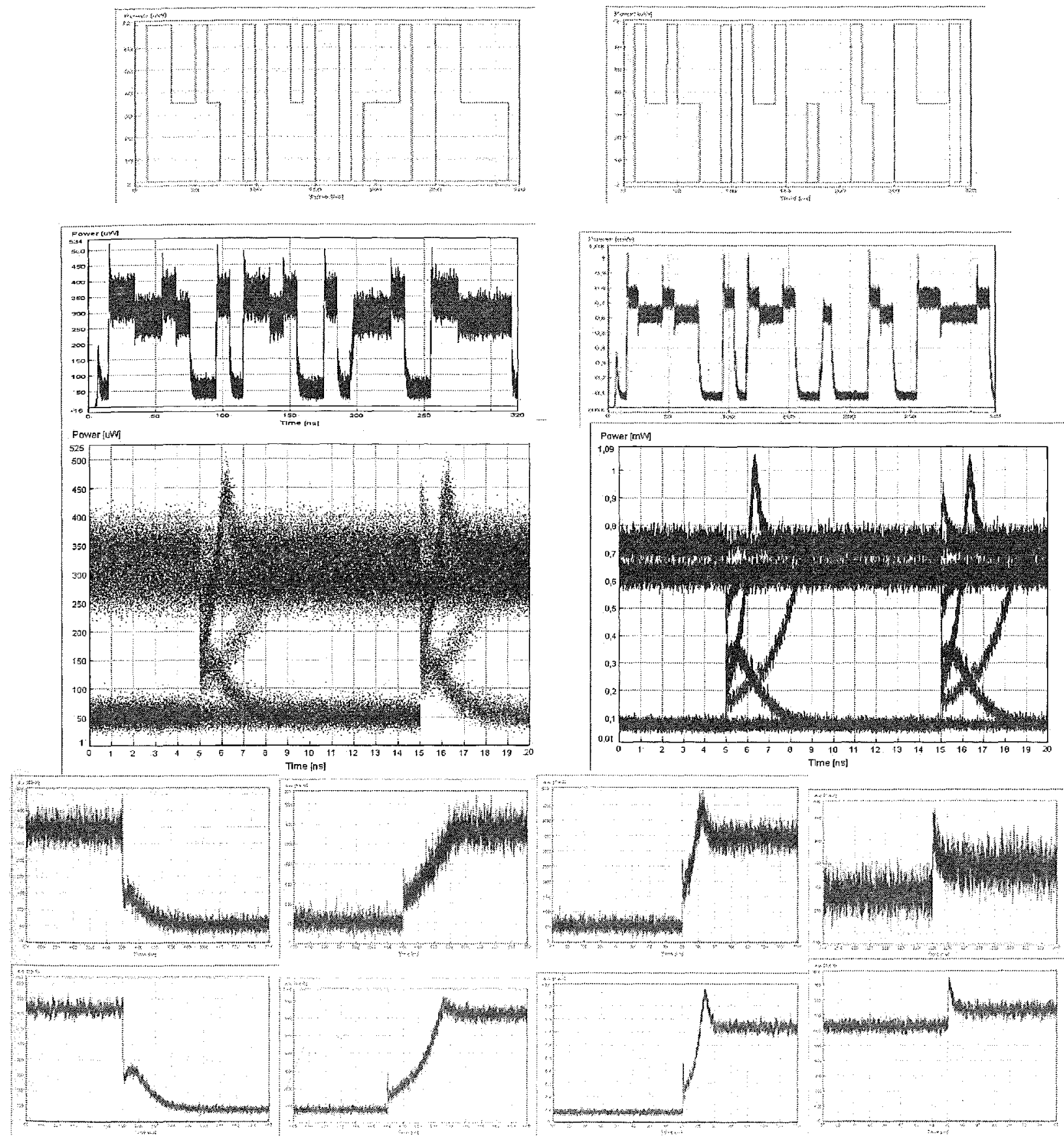

Figure 5. - First line of graphs shows the input data to the OLG. Second line the output of an OR function for the corresponding input data. Third line the eye diagram representation. Left column is for Q-device structure in Fabry-Perot and right column for DFB.

Two last lines: the one with higher noise correspond to FP_LD; last line to the DFB_LD. The detail representations of significantly transition are show: transition of input data from 1-0, 0-1,0-2,1-2. 
Figure 6 and 7 represent how the Q-device executes an ON- function. In this case we have not included the input signal because it does not give any additional information. We can have an idea of the input pattern from the output signal. Again the main noise is present from the input level near to the hysteresis cycle: transition from 1-2 and 2-1. Now we can see clearly the spike during switch-up and switch -down [9].
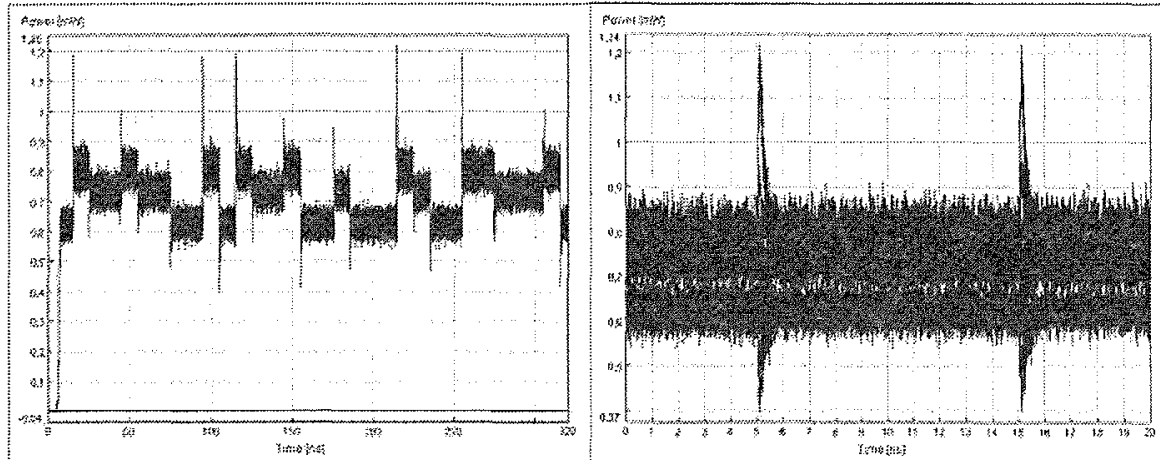

Figure 6. - First it is show the output of $\mathrm{Q}$-device with $\mathrm{h}=\mathrm{h}_{2}$. The eye diagram associated and the detail output for the two transitions more significant 1-2 and 2-1. DFB LD
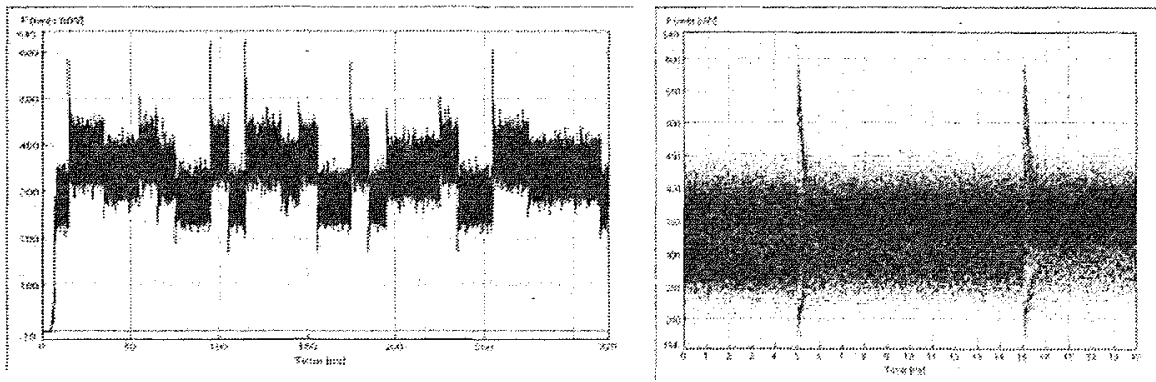

Figure 7. - First it is show the output of Q-device with $\mathrm{h}=\mathrm{h}_{2}$. The eye diagram associated and the detail output for the two transitions more significant 1-2 and 2-1. FP_ LD.

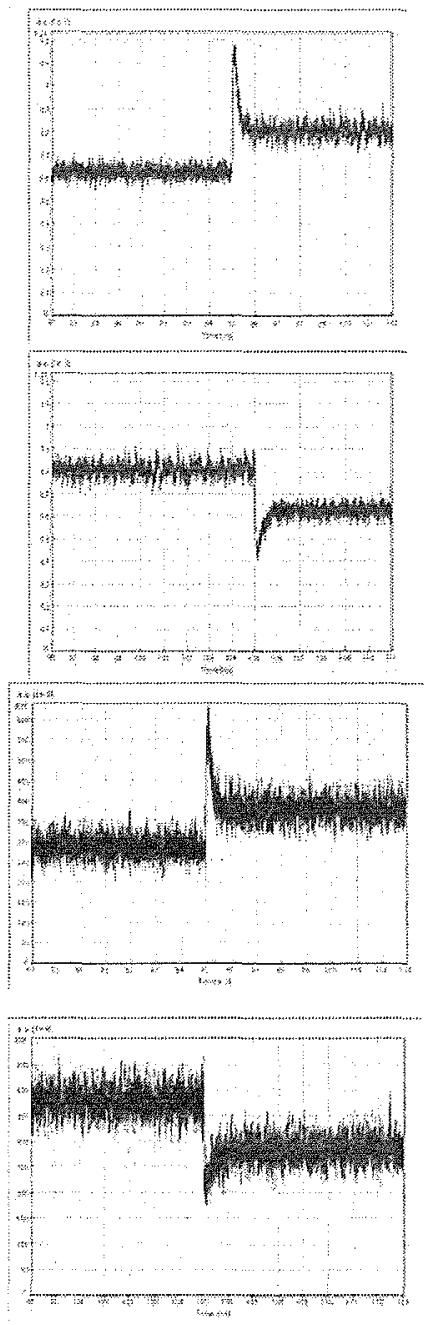

Output power from DFB is higher so it seems easier to discriminate the output. Here there is no eye pattern because there is only one output level with the ON function. There is an advantage with DFB amplifier: the potential for integration afforded by the removal of the requirement for lasers facets.

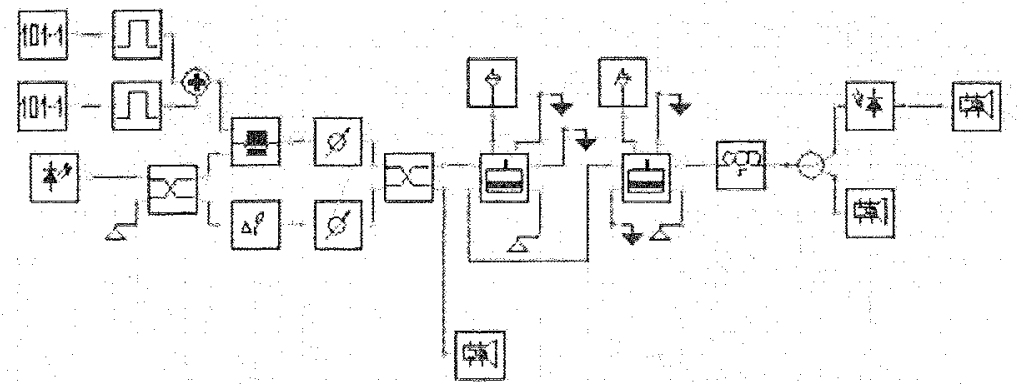

Figure 8. - Schematic of the model simulated by VPI_ComponentMaker ${ }^{T M}$ software tool for P-device.

\subsection{Simulation results for AND and $O R$ function}

On section 2 it was presented the transfer function of a P-device. This is a more complex device, based on SEED characteristics. Here it has been implemented with two FP-laser simulated with parameters of FP1 on reflection [13] and FP2 on transmission. It is not our intention to explain the model simulation here just to compare the AND and OR function with the eye pattern. 
Figure 9 represents the $\mathrm{OR}$ function that is obtained by applying a CW control signal equal to zero in P-device. If eye pattern is compared with those shown in figure 5 , it can be seen that greater noise is present for a zero.

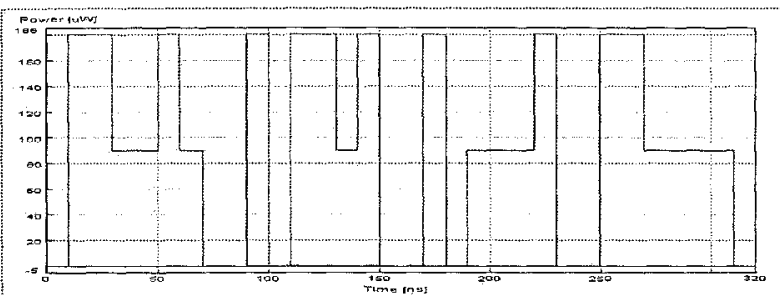

Figure 9. - On the right the input data to the OLG with a P-device. Second line the output of an OR function for the corresponding input data and the eye diagram representation.
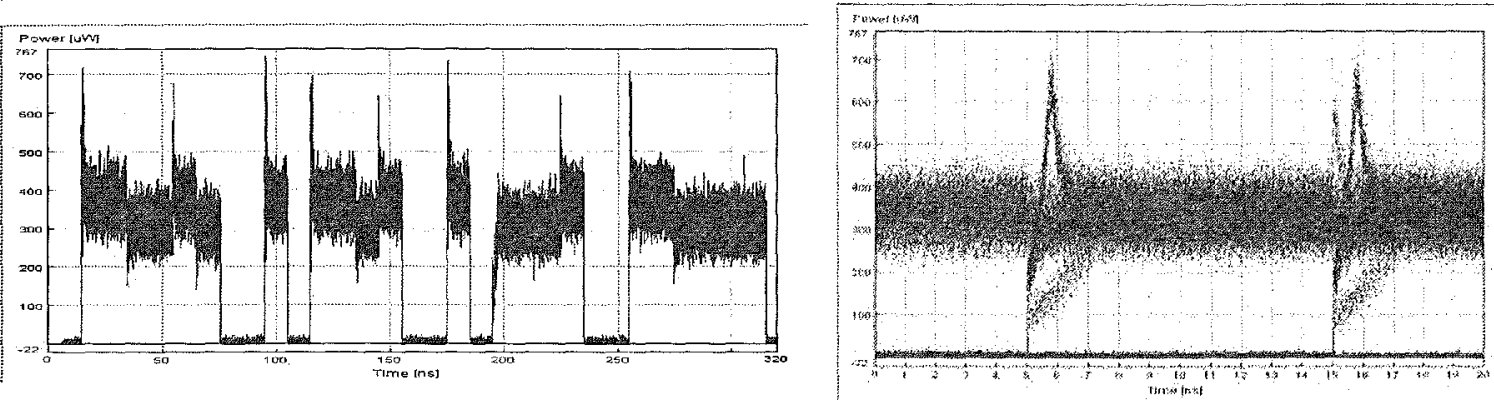

In the case of AND function it seems the opposite situation, the noise on zero value is higher with P-device. Function AND is obtained with a $\mathrm{CW}$ control signal $\mathrm{g}_{3}$, which means that device is working on the second valley of transfer function of figure 2. Again the signal level is near of the hysteresis cycle.

A further analyze of this P-device will allow better results.

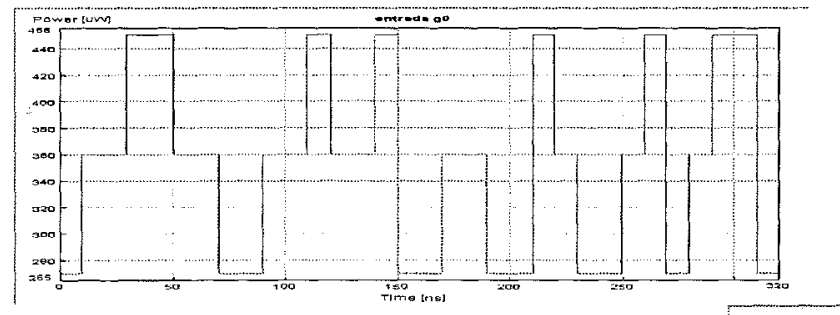

Figure 10. - On the right the input data to the OLG with a P-device. Second line the output of an AND function for the corresponding input data and the eye diagram representation.
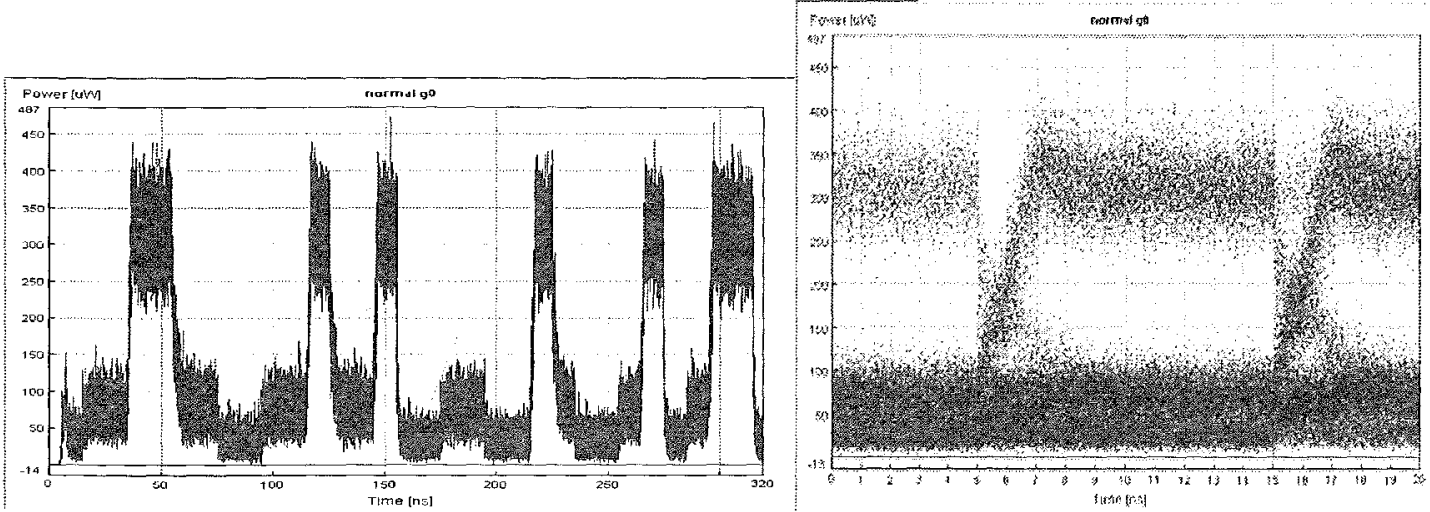
In all the cases the optical input signal has the same characteristics [14], the optical power for a bit ' 1 ' is always the same and also the central wavelength. The $\mathrm{CW}$ control signal must be with the same wavelengths as data signals, and the optical power depends on the function that it is required to be done by the device. Carefully requirements must be taken concerning optical signal phase but a deeper description of this problem is not of our intention here.

Certain relevant parameters used in the simulation are listed in Table I.

TABLE I

Laser Parameter for the Fabry-Perot and DFB Lasers used in the study.

\begin{tabular}{lccc}
\hline Laser Parameter & FP1 & FP2 & DFB \\
Cavity Length $(\mu \mathrm{m})$ & 350 & 400 & 300 \\
Left/Right Facet Reflectivity & 0.3 & 0.3 & - \\
Confinement Factor & 0.5 & 0.5 & 0.3 \\
Linear Material Gain Coeff. $\left(\mathrm{cm}^{2}\right)$ & $2.2 \cdot 10^{-16}$ & $2.2 \cdot 10^{-16}$ & $2.5 \cdot 10^{-16}$ \\
Linewidth Enhancement Factor & 6.9 & 6.9 & 5 \\
Linear Recombination Coeff. $(1 / \mathrm{s})$ & $1 \cdot 10^{8}$ & $1 \cdot 10^{8}$ & $1 \cdot 10^{8}$ \\
Bimolecular Recomb. Coeff. $\left(\mathrm{m}^{3} / \mathrm{s}\right)$ & $2 \cdot 10^{-16}$ & $2 \cdot 10^{-16}$ & $2 \cdot 10^{-16}$ \\
Auger Recombination Coeff. $\left(\mathrm{m}^{6} / \mathrm{s}\right)$ & $8.25 \cdot 10^{-41}$ & $8.25 \cdot 10^{-41}$ & $8.25 \cdot 10^{-41}$ \\
Transparency Carrier Density $\left(1 / \mathrm{m}^{3}\right)$ & $10^{24}$ & $10^{24}$ & $10^{24}$ \\
Fixed Internal Loss $(1 / \mathrm{m})$ & 5000 & 5000 & 1500 \\
Index Grating Coupling Coeff. $(1 / \mathrm{m})$ & - & - & 8000 \\
Bias current & $0.8 \cdot I_{\text {th }}$ & $0.88 \cdot I_{\text {th }}$ & $0.88 \cdot I_{\text {th }}$ \\
Frequency detuning (Ghz) & 30 & 15 & 14 \\
\hline
\end{tabular}

\section{CONCLUSIONS}

As it has been shown in previous paragraphs, the analysis of noise by the use of tools employed previously in optical communications, give the possibility to obtain some information about the behavior of logic cells under different conditions. As a matter of fact, "eye diagrams" offer the possibility to obtain certain initial and direct information about the response of the cell under noise conditions. It is evident that a first analysis should be possible without the use of this technique. But, as in optical communications, sometimes a previous and rapid interpretation of the final behavior of the system may be needed. The system will work under very different situations and under very different conditions. The easiest way to simulate this behavior is to inject a random signal to the system, with any possible combination of binary data, and to analyze the overall response. The best way to do that is, as we have shown, with the "eye diagram". This method has the main advantage of employing very well known techniques and, at the same time, the very sophisticated instrumentation employed in optical communications. No new methods or equipments are needed. This condition is as valid as the use of wavelengths employed in optical communications and no other optical frequencies, as for example those in the visible spectrum, employed in the first years of optical computing.

The method presented in this paper has allowed us to differentiate several regions in the switching behavior of the cells. A more detailed analysis would allow characterizing the importance that noise may have on the different regions of the logic cells time response. These aspects will be analyzed in the future.

\section{ACKNOWLEDGMENTS}

This work was partly supported by CICYT, grant TIC2003-04309, and CAM "Comunidad Autónoma de Madrid", grant FPI- Formación de Personal Investigador.

\section{REFERENCES}

1. A. González-Marcos \& J.A. Martin-Pereda, "Photonic switching architectures with logic cells" En:SPIE ITCom2003. Active Optical Transmission Systems and Equipment for WDM Networking II Paper 5246.82. Orlando, Florida USA Sept. 05-09. 2003

2. S. Haykin "Communication Systems" Wiley 2001 
3. A. González-Marcos and J. A. Martín-Pereda, "Analysis of irregular behavior on an optical computing logic cell", in Optics \& Laser Technology, Vol. 32, No 6, pp. 457-466. (2000).

4. A. González-Marcos and J. A. Martin-Pereda, "Method to analyze the influence of hysteresis in optical arithmetic units", in Optical Engineering, Vol. 40 No. 11, pp. 2371-2385. November 2001.

5. Emmanuel Desurvire "Erbium Doped-Fiber amplifiers. Principles and Applications" Wiley 2002.

6. A. Hurtado-Villavieja and A. González-Marcos. "An All-Optical Programmable Logic Gate with 1550nm Semiconductor Laser Amplifiers", to be presented in 'Optics in Computing 2004' the $21^{\text {st }}$ April 2004 in Engelberg (Switzerland).

7. A. González-Marcos and J. A. Martín-Pereda. "Digital chaotic output from an optically-processing element", in Optical Engineering, Vol. 35, pp. 525-535, (1996).

8. Linli Li, M.K. Haldar, C.Y Teo and F.V.C. Mendis "Dynamic properties of optically switched bistable semiconductor lasers", IEEE Journal of Quantum Electronics, Vol. 32, No. 6, pp. 1009-1014. June 1996.

9. M. J. Adams, 'Time-Dependent analysis of active and passive optical bistability in semiconductors', Proc. IEE, vol. 132, pt. J., pp.343-348, Dec. 1985.

10. Drew. N. Maywar and Govind P. Agrawal, "Transfer-Matrix Analysis of Optical Bistability in DFB Semiconductor Laser Amplifiers with Nonunifor Gratings", IEEE Journal of Quantum Electronics, Vol. 33, No. 11, pp. 2029-2037. November 1997.

11. Drew. N. Maywar and Govind P. Agrawal, "Effect of Chirped Gratings on Reflective Optical Bistability in DFB Semiconductor Laser Amplifiers", IEEE Journal of Quantum Electronics, Vol. 34, No. 12, pp. 2364-2370. December 1998.

12. M. J. Adams, R. Wyatt, "Optical Bistability in Distributed Feedback Semiconductor Laser Amplifiers", in IEE Proceedings Pt. J Optoelectronics, Vol. 134, No. 1, pp. 58-63. February 1987.

13. P. Pakdeevanich and M. J. Adams, "Measurements and Modeling of Reflective Bistability in 1.55 4am Laser Diode Amplifiers", IEEE Journal of Quantum Electronics, Vol. 35, No. 12, pp. 1894-1903. December 1999.

14. Zeqi Pan, Hongchin Lin, and M. Dagenais, "Switching Power Dependence on detuning and current in bistable diode laser amplifiers", in Appl. Phys. Lett. Vol. 58, No. 7, pp. 687-689. February 1991. 